\title{
Linear response in theory of electron transfer reactions as an alternative to the molecular harmonic oscillator model
}

\author{
Yuri Georgievskii, Chao-Ping Hsu, and R. A. Marcus \\ Noyes Laboratory of Chemical Physics, Mail Code 127-72, California Institute of Technology, \\ Pasadena, California 91125
}

(Received 21 October 1998; accepted 16 December 1998)

\begin{abstract}
The effect of solvent fluctuations on the rate of electron transfer reactions is considered using linear response theory and a second-order cumulant expansion. An expression is obtained for the rate constant in terms of the dielectric response function of the solvent. It is shown thereby that this expression, which is usually derived using a molecular harmonic oscillator ("spin-boson") model, is valid not only for approximately harmonic systems such as solids but also for strongly molecularly anharmonic systems such as polar solvents. The derivation is a relatively simple alternative to one based on quantum field theoretic techniques. The effect of system inhomogeneity due to the presence of the solute molecule is also now included. An expression is given generalizing to frequency space and quantum mechanically the analogue of an electrostatic result relating the reorganization free energy to the free energy difference of two hypothetical systems [J. Chem. Phys. 39, 1734 (1963)]. The latter expression has been useful in adapting specific electrostatic models in the literature to electron transfer problems, and the present extension can be expected to have a similar utility. (C) 1999 American Institute of Physics. [S0021-9606(99)01511-1]
\end{abstract}

\section{INTRODUCTION}

Interaction with an environment plays a crucial role in many nonadiabatic processes in condensed phases. Electron transfer reactions ${ }^{1-3}$ provide a major example in which strong electrostatic interaction of a reacting species with a polar solvent can control both the energetics and the dynamics of the process. The theory of nonadiabatic transitions, in the presence of strong interaction with the environment treated quantum mechanically, originates from the papers of $\mathrm{Lax}^{4}$ and Kubo and Toyozawa. ${ }^{5}$ Their approach was applied by Levich and Dogonadze ${ }^{6}$ to calculate the rate of an electron transfer reaction in liquids. In their theory Levich and Dogonadze, like Lax and Kubo and Toyozawa, used a collection of harmonic oscillators as a model of the environment whose fluctuations are responsible for the electronic transition.

In a seemingly different approach Marcus $^{7-9}$ used a dielectric continuum approach and showed that the polarity of the solvent is important for understanding the energetics of an ET reaction. Marcus later assumed only a linear dielectric response of the solvent in the vicinity of the reacting species, and did not use any specific molecular model of the solvent. ${ }^{9}$ Vorotyntsev et al. ${ }^{10}$ related the rate constant to a complex, space- and time-dependent electric susceptibility $\epsilon(\omega, k)$ in the framework of a harmonic oscillator model, thus generalizing Marcus' theory in this respect, which was purely classical. Jortner and co-workers ${ }^{11-13}$ extended the treatment ${ }^{7}$ of Marcus and Levich and Dogonadze ${ }^{6}$ by using the former for low frequency modes and the latter for high frequency modes.

One factor influencing the dielectric properties of polar solvents is the reorientational motion of the solvent molecules, a motion which is strongly anharmonic. Thus, the approach of Levich and Dogonadze, subsequently used as a common model to treat quantum aspects of ET reactions, ${ }^{14,15}$ required justification. One way to resolve this difficulty was given by Ovchinnikov and Ovchinnikova, ${ }^{16}$ who used a quantum field theoretic method ${ }^{17}$ to show that the result of Levich and Dogonadze can be recovered in the long-wave approximation without explicitly invoking a molecular harmonic oscillator model. In their derivation Ovchinnikov and Ovchinnikova considered uniform systems, so dielectric image effects and any effect of the solute on the properties of the nearby solvent were not included. Image effects have been included earlier ${ }^{7-9}$ on the assumption that the slow and fast solvent modes allow for a clear separation.

Kornyshev investigated the models with nonlocal dielectric response, ${ }^{18-20}$ which was applied earlier to electron transfer reactions in the framework of a molecular harmonic oscillator model. ${ }^{14,21,22}$ Song, Chandler, and Marcus ${ }^{23}$ considered the effect of the solvent inhomogeneity due to the solute presence using the Gaussian field model, which could be viewed as a continuum equivalent of a molecular harmonic oscillator model. Fleming and co-workers ${ }^{24-26}$ used nonlinear spectroscopy to study the solvation dynamics of chromophoric molecules in the variety of solvents and glasses. They related the spectral density of the harmonic modes to the correlation function of the energy difference in the excited and the ground electronic states of the chromophore and used a treatment developed by $\mathrm{Mukamel}^{27}$ to express the measured spectra in tems of the spectral density of the effective harmonic oscillators. Berne and co-workers ${ }^{28}$ have investigated the solvation dynamics and vibronic spectra of chromophoric molecules for models in which the harmonic bath frequencies and normal modes are different, rather than the same, for the initial and final electronic states. 
In this paper we show that an expression for the rate constant in terms of dielectric dispersion properties, which was originally derived using a molecular harmonic oscillator model,${ }^{10,14}$ is also applicable to molecular systems which are strongly anharmonic, in particular to polar solvents. The present calculation is based on linear response theory ${ }^{29}$ and a second-order cumulant ${ }^{29,30}$ expansion. The calculation then relates the rate constant to the dielectric and some structural properties of the system. An expression is also obtained [Eqs. (17), (23), and (38)] generalizing to frequency space and in quantum mechanical terms a result obtained earlier. ${ }^{31,32}$ The latter related the free energy barrier for reaction to a difference in "free energy" of two hypothetical systems. These two systems have a charge distribution which is the difference between those of the products' and the reactants', but now one system interacts via the solvent dielectric dispersion at frequency $\omega$ and the other at the solvent's optical frequency.

The cumulant expansion approach is the same as one developed by $\mathrm{Kubo}^{29}$ and Mukamel ${ }^{33}$ to describe the transient fluorescence of large aromatic molecules. A similar approach was used earlier by Hizhnyakov and co-workers ${ }^{34-36}$ to describe the spectra of impurity centers in solids. There is perhaps also a pedagogical interest in such a treatment, since it provides a simplification and extension over the original pioneering and stimulating quantum field theoretic ${ }^{17}$ derivation of Ovchinnikov and Ovchinnikova. ${ }^{16}$ Another alternative approach to the present one is that given by Chandler, who used a path integral formulation. ${ }^{15}$ The common harmonic oscillator bulk model can be viewed as one way of visualizing these more rigorous results.

The paper is organized as follows:

The molecular Hamiltonian and the molecular solvent polarization operator $\hat{\mathbf{P}}_{0}(\mathbf{r})$ are described in Sec. II A. In Sec. II B the standard expression for the nonadiabatic reaction rate constant is given and a generalized coordinate $X$ is introduced. (Use of the latter provides a way of avoiding the field theoretical treatment.) The correlation function in the rate constant expression is simplified using a second-order cumulant expansion and a correlation function of $X$. Linear response theory is introduced in Sec. IIC to evaluate this correlation function. The $X$ is then related in Sec. II D to the solvent polarization operator $\hat{\mathbf{P}}_{0}(\mathbf{r})$ and to a certain hypothetical electric field $\mathbf{D}_{12}^{(0)}(\mathbf{r})$ and is used to evaluate the solvent response function $\alpha_{\omega}$. The $\alpha_{\omega}$ is expressed in Sec. IIE in terms of a frequency-dependent "free energy" difference of two hypothetical systems, Eq. (38), and the equation for the rate constant is then given [Eq. (39)]. Some specific solute models are discussed in Sec. IIF. The relation of the present results to previous work and some other features of the results are considered in the Discussion, Sec. III. Several features are treated in more detail in the appendices: The various electrostatic operators are treated in Appendix A, the relation of a term in the rate expression to the standard free energy of reaction is derived in Appendix B, and the relation between the electrostatic operators and the time-dependent macroscopic properties is given in Appendix C. In Appendix $\mathrm{D}$ it is shown how the treatment of the excluded volume effect in the present formulation is related to the one of Ref. 23.

\section{THEORY}

\section{A. Hamiltonian and molecular polarization operator}

The Hamiltonian operator of the system reactants + solvent in either electronic state can be written as

$$
H_{j}=h_{j}+H_{0}+H_{j}^{\prime}, \quad j=1,2,
$$

where $h_{j}$ is the Hamiltonian of the "free reactants," $H_{0}$ is the Hamiltonian of the "free solvent," and $H_{j}^{\prime}$ is the interaction between them. The $H_{j}^{\prime}$ depends on the electronic state of the reactants. The Hamiltonian $h_{j}$ includes the intramolecular vibrational modes of the reactants. Purely for simplicity of notation, since the present article focuses on the solvent effect which is associated with $H_{j}^{\prime}$ and $H_{0}$, we consider structureless reactants and so treat $h_{j}$ as just numbers. The role of the intramolecular vibrational modes has been extensively discussed in the literature ${ }^{12,13,37-39}$ and can be easily incorporated into the present scheme. The operators $H_{0}$ and $H_{j}^{\prime}$ act on the nuclear wave function of the solvent. We do not use carets in their notation in contrast with other operators [e.g., in Eq. (2) below] since they are used only in this sense and so no ambiguity is caused by such notation.

The interaction between the reacting species and the solvent is approximately separated into electrostatic and nonelectrostatic interactions. Nonelectrostatic interaction includes van der Waals attraction, short range repulsion, and hydrogen bonding. This interaction is assumed here to be independent of the electronic state of the solute and is included in $H_{0}$. However, it should be noted that the last assumption probably breaks down for the reactions in which a bond of the solute to a solvent molecule (including a hydrogen bond) is formed or broken when the electronic state of the reacting species changes. Such an effect could probably be included later, instead, as a state-specific contribution to $h_{j}$.

Because of the complex interactions of dipoles, permanent and induced, of the solvent there are many subtleties in their analysis. In an excellent article written in 1958, Mandel and Mazur ${ }^{40}$ have described these terms as well as previous errors in the literature. The electrostatic interaction between the reactants and the solvent can be written, as shown in Appendix A [cf. Eq. (A6)], as ${ }^{16}$

$$
H_{j}^{\prime}=-\int \mathbf{D}_{j}^{(0)}(\mathbf{r}) \cdot \hat{\mathbf{P}}_{0}(\mathbf{r}) d^{3} \mathbf{r}, \quad j=1,2,
$$

where $\mathbf{D}_{j}^{(0)}(\mathbf{r})$ is the electric field at point $\mathbf{r}$, created by the reacting species in vacuum for the $j$ th electronic state and $\hat{\mathbf{P}}_{0}(\mathbf{r})$ is the operator representing the molecular solvent polarization at the same point, when the solute charge distribution $\rho(\mathbf{r})$ is set equal to zero. We use a caret notation for $\hat{\mathbf{P}}_{0}(\mathbf{r})$ to stress that it is an operator acting on the nuclear wave function of the solvent. Although we do not explicitly use the following expression for $\hat{\mathbf{P}}_{0}(\mathbf{r})$ in Eq. (2) it could be defined in a long-wave approximation as a weighted sum of the individual dipole moments of the solvent molecules: 


$$
\hat{\mathbf{P}}_{0}(\mathbf{r})=\sum_{n} \delta\left(\mathbf{r}-\hat{\mathbf{r}}_{n}\right) \hat{\mathbf{d}}_{n} \equiv \sum_{n} \delta\left(\mathbf{r}-\hat{\mathbf{r}}_{n}\right)\left(\hat{\mathbf{d}}_{n}^{0}+\hat{\mathbf{d}}_{n}^{\prime}\right) .
$$

In this equation the coordinates of the center of mass of the $n$th solvent molecule are denoted by $\hat{\mathbf{r}}_{n}$ and its net dipole moment by $\hat{\mathbf{d}}_{n}$. This $\hat{\mathbf{d}}_{n}$ is not only a function of the internal nuclear configuration of the $n$th solvent molecule but also of the other solvent molecules, since they induce an electronic polarization in it. As a result, the solvent polarization operator $\hat{\mathbf{P}}_{0}(\mathbf{r})$ itself consists of two parts, one part arising from all the unperturbed dipole operators $\hat{\mathbf{d}}_{n}^{0}$ of the solvent molecules, and the second part related to $\hat{\mathbf{d}}_{\mathbf{n}}^{\prime}$ being a collective electronic response to the first part.

$\hat{\mathbf{r}}_{n}$ and $\hat{\mathbf{d}}_{n}$ are quantum mechanical operators which act on the wave function in the configurational space of all nuclear degrees of freedom of the solvent, after an averaging over the total electronic wave function of the solvent has been performed. This averaging assumes a fast electronic response. It may be stressed that no averaging over any nuclear motion of the solvent, such as over the intermolecular or intramolecular vibrations of the solvent molecules, is performed in Eqs. (2) and (3). Instead, the molecular solvent polarization operator $\hat{\mathbf{P}}_{0}(\mathbf{r})$ is a complicated function of all nuclear coordinates of the solvent. $\hat{\mathbf{P}}_{0}(\mathbf{r})$ is a part of the total polarization operator $\hat{\mathbf{P}}(\mathbf{r})=\hat{\mathbf{P}}_{0}(\mathbf{r})+\mathbf{P}_{\mathrm{op}}(\mathbf{r})$ [cf. Eq. (C4)], which also includes the electronic response $\mathbf{P}_{\mathrm{op}}(\mathbf{r})$ to the solute charge distribution. The total polarization operator $\hat{\mathbf{P}}(\mathbf{r})$ is distinguished from the standard ${ }^{41}$ macroscopic polarization $\mathbf{P}(\mathbf{r})$, which is an average of $\hat{\mathbf{P}}(\mathbf{r})$ over local solvent nuclear fluctuations [cf. Eq. (C1)].

Since the molecular solvent polarization operator $\hat{\mathbf{P}}_{0}(\mathbf{r})$ in Eq. (2) was defined in Eq. (3) using a hypothetical neutral state of the solute with a charge distribution $\rho(\mathbf{r})=0$, it is, therefore, a function of the solvent state only. In particular, it does not depend on the electronic state of the solute. In Appendix $\mathrm{A}$ it is shown that the expression for the electrostatic interaction between the solute and the solvent, given by Eq. (2), is valid under rather general conditions.

\section{B. Rate constant in terms of correlation function of a generalized coordinate $X$}

The Hamiltonians $H_{j}, j=1,2$, describe the collective motion of the system in the initial (reactant) and final (product) electronic states of the solute. To describe the transition between the two one must introduce the nondiagonal matrix element $\Delta$ which couples the two electronic states. This matrix element is sometimes small and is assumed to be independent of the nuclear configuration for statistically important configurations (Condon approximation). Non-Condon effects could be of importance for solvated electrons and solutes with weekly localized electronic clouds, ${ }^{42,43}$ but not for tight redox couples. Then, in first-order perturbation theory the rate constant $k$ for a nonadiabatic transition between the two electronic states of a reactant or reactants fixed in position can be written as ${ }^{5}$

$$
k=\Delta^{2} \int_{-\infty}^{+\infty} d t\left\langle e^{i t H_{1}} e^{-i t H_{2}}\right\rangle,
$$

(throughout units in which $\hbar=1$ are used), where the averaging is taken over the initial state:

$$
\langle\cdots\rangle=\frac{\operatorname{Tr}\left[\cdots e^{-\beta H_{1}}\right]}{\operatorname{Tr}\left[e^{-\beta H_{1}}\right]}, \quad \beta=1 / k_{B} T .
$$

It is convenient to rewrite the expression for the correlation function in Eq. (4) in terms of the formalism of timeordered exponents: ${ }^{4}$

$$
e^{i t H_{1} t} e^{-i t H_{2}}=e_{+}^{-i \int_{0}^{t} X(\tau) d \tau},
$$

where

$$
X=H_{2}-H_{1}
$$

is the energy difference of the system in the final and initial electronic states of the solute (the nuclear kinetic energy operator cancels in $X$ ) and where the time-evolution occurs in the initial state,

$$
X(\tau)=\exp \left(i \tau H_{1}\right) X \exp \left(-i \tau H_{1}\right) .
$$

One can then use a cumulant expansion ${ }^{29}$ to represent the correlation function $\left\langle e^{i t H_{1}} e^{-i t H_{2}}\right\rangle$ :

$$
\begin{aligned}
\left\langle e^{i t H_{1}} e^{-i t H_{2}}\right\rangle=\exp & \left\{\sum_{n=1}^{\infty} \frac{(-i)^{n}}{n !} \int_{-\infty}^{+\infty} \cdots \int_{-\infty}^{+\infty} d \tau_{1} \cdots d \tau_{n}\right. \\
& \left.\times\left\langle\mathrm{T}\left[X\left(\tau_{1}\right), \cdots, X\left(\tau_{n}\right)\right]\right\rangle_{c}\right\},
\end{aligned}
$$

where $T[\cdots]$ denotes the time-ordering operator and the average $\langle\cdots\rangle_{c}$ in the $n$th term of the power of exponent denotes the cumulant of the $n$th order. As an approximation, using a cumulant expansion and retaining only the terms up to second order, we have $e^{30}$

$$
\left\langle e^{i t H_{1}} e^{-i t H_{2}}\right\rangle=\exp \left\{-i t\langle X\rangle-\int_{0}^{t} d \tau_{1} \int_{0}^{\tau_{1}} d \tau_{2} \quad C\left(\tau_{1}-\tau_{2}\right)\right\},
$$

where

$$
C(\tau)=\langle\delta X(\tau) \delta X(0)\rangle,
$$

and $\delta X=X-\langle X\rangle$ is the variation of $X$ from its average value $\langle X\rangle$ in the initial state. The expressions for the first two cumulants were used:

$\langle X\rangle_{c}=\langle X\rangle$,

$\langle X(\tau) X(0)\rangle_{c}=\langle X(\tau) X(0)\rangle-\langle X\rangle^{2}=\langle\delta X(\tau) \delta X(0)\rangle$.

Equation (10) shows that in this approximation the correlation function $\left\langle e^{i t H_{1}} e^{-i t H_{2}}\right\rangle$ is expressed in terms of the correlation function $C(t)$. In a molecular harmonic oscillator model the higher cumulants vanish exactly ${ }^{4,5}$ if $X$ is a linear combination of the normal modes and Eq. (10) then becomes exact for that model.

To express Eq. (11) in terms of a spectral density, namely Eq. (15) below, a Fourier transform $\widetilde{C}(\omega)$ is introduced, 


$$
\widetilde{C}(\omega)=\int_{-\infty}^{+\infty} C(t) e^{i \omega t} d t
$$

which is a real and positive function of $\omega$. It can be shown that $\widetilde{C}(\omega)$ satisfies the relation

$$
\widetilde{C}(\omega)=e^{\omega \beta} \widetilde{C}(-\omega) .
$$

The last equation is a direct consequence of the fluctuationdissipation theorem. ${ }^{29}$

The spectral density $J(\omega)$ can be defined by

$$
J(\omega)=\frac{1}{2}[\widetilde{C}(\omega)-\widetilde{C}(-\omega)] .
$$

The following standard expression for the correlation function $C(t)$ is then readily obtained:

$$
C(t)=\frac{1}{\pi} \int_{0}^{\infty} d \omega J(\omega)[\operatorname{coth}(\beta \omega / 2) \cos \omega t-i \sin \omega t] .
$$

From Eqs. (4), (10), and (16) a standard expression for the nonadiabatic reaction rate ${ }^{5,6,14,16}$ follows:

$$
\begin{aligned}
k= & \Delta^{2} \int_{-\infty}^{\infty} d t \exp \left[-i t \Delta G^{0}\right. \\
& \left.+\frac{1}{\pi} \int_{0}^{\infty} d \omega \frac{J(\omega)}{\omega^{2}} \frac{\cosh (\beta \omega / 2-i \omega t)-\cosh (\beta \omega / 2)}{\sinh (\beta \omega / 2)}\right],
\end{aligned}
$$

where $\Delta G^{0}$ is shown in Appendix B to be the standard free energy of reaction.

\section{Relation of the correlation function of $X, C(t)$, to the generalized susceptibility $\alpha_{\omega}$}

The correlation function $C(t)$ for the anharmonic molecular system is next expressed in terms of the dielectric properties of the solvent. To this end linear response theory is used, after introducing an effective Hamiltonian $H_{\text {eff }}(t)$ :

$$
H_{\text {eff }}(t)=H_{1}-X f(t) \text {. }
$$

Here, the energy difference $X$ plays the role of a generalized coordinate (reaction coordinate) and $f(t)$ is a generalized dimensionless force. Thereby, $H_{\text {eff }}$ can evolve from $H_{1}$ to $H_{2}$, if $f(t)$ is chosen to tend to zero as $t \rightarrow-\infty$ and to become unity as $t \rightarrow+\infty$.

A nonequilibrium density matrix $R(t)$ which evolves according to a Liouville equation with the Hamiltonian $H_{\text {eff }}(t)$, Eq. (18), is next introduced,

$$
\frac{d}{d t} R(t)=-i\left[H_{\mathrm{eff}}(t), R(t)\right],
$$

and used to define a time-dependent average $\bar{X}(t)$ at time $t$,

$$
\bar{X}(t)=\operatorname{Tr}[X R(t)] .
$$

In linear response theory, the average value of the generalized coordinate $\bar{X}(t)$ is linearly related to the generalized force: ${ }^{29}$

$$
\bar{X}(t)-\langle X\rangle=\delta \bar{X}(t)=\int_{0}^{\infty} \alpha(t) f(t-\tau) d \tau,
$$

where the generalized susceptibility $\alpha(\tau)$ is given in terms of a commutator of $X(\tau)$ and $X(0)^{29}$

$$
\alpha(\tau)=\langle[\delta X(\tau), \delta X(0)]\rangle=-2 \operatorname{Im} C(\tau) .
$$

The second part of Eq. (22) follows directly from the definition of the correlation function $C(\tau)$ in Eq. (11). In passing, we note that we have been setting $\hbar=1$. With ordinary units of $\hbar$, the left side of Eq. (22) would read $\alpha(\tau) \hbar$.

Comparing Eqs. (15), (16), and (22) the spectral density $J(\omega)$ is seen to coincide with the negative imaginary part of the Fourier component of the linear response function $\alpha_{\omega}$ $=\int_{0}^{\infty} \alpha(t) \exp (i \omega t) d t$ :

$$
J(\omega)=-\operatorname{Im} \alpha_{\omega} .
$$

\section{Relation of $C(t)$ and $J(\omega)$ to dielectric properties}

$C(t)$ and $J(\omega)$ are molecular statistical mechanical properties. We turn next to their calculation in terms of the dielectric properties of the solvent, in the long wavelength (local response) approximation for the solvent. Upon substituting Eqs. (1) and (2) into Eq. (7) the following expression for the reaction coordinate $X$ is obtained:

$$
X=-\int \mathbf{D}_{21}^{(0)}(\mathbf{r}) \cdot \hat{\mathbf{P}}_{0}(\mathbf{r}) d^{3} \mathbf{r},
$$

where $\mathbf{D}_{21}^{(0)}$ is the difference of the vacuum electric fields in the initial and final states of the solute,

$$
\mathbf{D}_{21}^{(0)}(\mathbf{r})=\mathbf{D}_{2}^{(0)}(\mathbf{r})-\mathbf{D}_{1}^{(0)}(\mathbf{r}) .
$$

Equations (18) and (24) yield

$$
H_{\text {eff }}=H_{1}-\int \mathbf{D}^{(0)}(\mathbf{r}, t) \cdot \hat{\mathbf{P}}_{0}(\mathbf{r}) d^{3} \mathbf{r},
$$

where

$$
\mathbf{D}^{(0)}(\mathbf{r}, t)=\mathbf{D}_{21}^{(0)}(\mathbf{r}) f(t),
$$

is the vacuum electric field formed by the external, timedependent charge distribution $\rho(\mathbf{r}, t)$. The latter is a linear combination of the charge distributions of the solute in its initial and final electronic states:

$$
\rho(\mathbf{r}, t)=\rho_{21}(\mathbf{r}) f(t), \quad \rho_{21}(\mathbf{r})=\rho_{2}(\mathbf{r})-\rho_{1}(\mathbf{r}) .
$$

The effective Hamiltonian (26) describes the interaction of the solvent with the external charge distribution $\rho(\mathbf{r}, t)$. Thus, the calculation of the correlation function $C(t)$ is reduced to finding the linear dielectric response of the solvent to the time-dependent external electric field $\mathbf{D}^{(0)}(\mathbf{r}, t)$ given by Eq. (27). ${ }^{44}$ The standard "macroscopic" treatment can then be used to calculate the average electric field $\mathbf{E}(\mathbf{r}, t)$ and the average electric displacement $\mathbf{D}(\mathbf{r}, t)$ which arise in the solvent in response to the external time-dependent electric field $\mathbf{D}^{(0)}(\mathbf{r}, t)$. In Appendix $C$ it is shown how $\mathbf{E}(\mathbf{r}, t)$ and $\mathbf{D}(\mathbf{r}, t)$ are related to the solvent polarization operator $\hat{\mathbf{P}}_{0}(\mathbf{r})$ in terms of the molecular model of the solvent. These fields $\mathbf{E}(\mathbf{r}, t)$ and $\mathbf{D}(\mathbf{r}, t)$ coincide with the corresponding macroscopical fields in the standard electrostatic and electrodynamic treatments. ${ }^{41,45}$

As a result the standard electrostatic equations for $\mathbf{E}(\mathbf{r}, t)$ and $\mathbf{D}(\mathbf{r}, t)$ can be used: ${ }^{45}$ 
$\nabla \cdot \mathbf{D}(\mathbf{r}, t)=4 \pi \rho(\mathbf{r}, t), \quad \nabla \times \mathbf{E}(\mathbf{r}, t)=0$.

$\mathbf{E}(\mathbf{r}, t)$ and $\mathbf{D}(\mathbf{r}, t)$ are coupled via the solvent electric susceptibility $\epsilon\left(\mathbf{r}, \mathbf{r}^{\prime} \tau\right)$, which is generally nonlocal in both the space and time domains,

$$
\mathbf{D}(\mathbf{r}, t)=\int_{0}^{\infty} d \tau \int d \mathbf{r}^{\prime} \epsilon\left(\mathbf{r}, \mathbf{r}^{\prime}, \tau\right) \mathbf{E}(\mathbf{r}, t-\tau) .
$$

We will use a standard, long-wave approximation for $\epsilon\left(\mathbf{r}, \mathbf{r}^{\prime}, \tau\right)$ in which

$$
\epsilon\left(\mathbf{r}, \mathbf{r}^{\prime}, \tau\right) \simeq \epsilon(\mathbf{r}, \tau) \delta^{3}\left(\mathbf{r}-\mathbf{r}^{\prime}\right),
$$

where $\delta^{3}(\mathbf{r})$ is the three-dimensional delta-function.

One feature of Eqs. (29)-(31) is the inhomogeneity of the dielectric environment reflected in $\epsilon(\mathbf{r}, \tau)$, since the Hamiltonian $H_{1}$ includes the nonelectrostatic interaction between the solvent and the reacting species which defines the structural properties of the solvent in the vicinity of the reactants.

The solution of Eqs. (29)-(31) is facilitated by taking the time-Fourier transform and so going over to the frequency domain. As a result, Eqs. (29)-(31) are reduced to electrostatic-like equations in which the usual static electric susceptibility $\epsilon_{0}$ is replaced by its frequency analog $\epsilon_{\omega}(\mathbf{r})$ $=\int_{0}^{\infty} \epsilon(\mathbf{r}, \tau) \exp (i \omega \tau) d \tau$

$$
\begin{aligned}
& \nabla \cdot \mathbf{D}_{\omega}(\mathbf{r})=4 \pi \rho_{21}(\mathbf{r}), \quad \nabla \times \mathbf{E}_{\omega}(\mathbf{r})=0, \\
& \mathbf{D}_{\omega}(\mathbf{r})=\boldsymbol{\epsilon}_{\omega}(\mathbf{r}) E_{\omega}(\mathbf{r}),
\end{aligned}
$$

where $\mathbf{E}_{\omega}(\mathbf{r})$ and $\mathbf{D}_{\omega}(\mathbf{r})$ denote the corresponding Fourier components of the electric field and electric displacement per unit $f_{\omega}$ :

$$
\begin{aligned}
& \mathbf{E}_{\omega}(\mathbf{r})=\frac{1}{f_{\omega}} \int_{-\infty}^{\infty} \mathbf{E}(\mathbf{r}, t) \exp (i \omega t) d t, \\
& \mathbf{D}_{\omega}(\mathbf{r})=\frac{1}{f_{\omega}} \int_{-\infty}^{\infty} \mathbf{D}(\mathbf{r}, t) \exp (i \omega t) d t .
\end{aligned}
$$

Here, $f_{\omega}=\int_{-\infty}^{\infty} f(t) \exp (i \omega t) d t$ is the Fourier component of the generalized force $f(t)$ [cf. Eq. (21)]. $\mathbf{E}_{\omega}(\mathbf{r})$ and $\mathbf{D}_{\omega}(\mathbf{r})$ so defined are independent of the function $f(t){ }^{46}$

The spatial inhomogeneity of the solvent enters into Eqs. (32) via the spatial dependence of $\epsilon_{\omega}(\mathbf{r})$. In the presence of any sharp boundaries in the problem, the appropriate boundary conditions for the electric field $\mathbf{E}_{\omega}(\mathbf{r})$ and electric displacement $\mathbf{D}_{\omega}(\mathbf{r})$ must be used to solve Eqs. (32).

Using the expression for the reaction coordinate $X$, Eq. (24), and Eqs. (C4)-(C10), the following expression for the Fourier component of the averaged reaction coordinate, $\delta \bar{X}_{\omega}=\int_{-\infty}^{\infty} \delta \bar{X}(t) \exp (i \omega t) d t$, is readily obtained:

$$
\delta \bar{X}_{\omega}=-f_{\omega} \int \mathbf{D}_{21}^{(0)}(\mathbf{r}) \cdot\left[\mathbf{P}_{\omega}(\mathbf{r})-\mathbf{P}_{\mathrm{op}}(\mathbf{r})\right] d^{3} \mathbf{r},
$$

where $\mathbf{P}_{\omega}$ is the Fourier component of the total average solvent polarization, Eq. (C10), and $\mathbf{P}_{\mathrm{op}}(\mathbf{r})$ is a part of the electronic polarization which is induced by the external charge distribution, Eq. (C5). The difference $\mathbf{P}_{\omega}(\mathbf{r})-\mathbf{P}_{\mathrm{op}}(\mathbf{r})$ reflects the induced nuclear polarization of the solvent. $\mathbf{P}_{\omega}(\mathbf{r})$ tends to $\mathbf{P}_{\text {op }}$ when $\omega$ approaches the optical frequency region.
On comparing Eqs. (34) and (21) an expression follows for the Fourier component of the linear response function $\alpha_{\omega}=\int_{0}^{\infty} \alpha(t) \exp (i \omega t) d t$ :

$\alpha_{\omega}=\int \mathbf{D}_{21}^{(0)}(\mathbf{r}) \cdot \mathbf{P}_{\mathrm{op}}(\mathbf{r}) d^{3} \mathbf{r}-\int \mathbf{D}_{21}^{(0)}(\mathbf{r}) \cdot \mathbf{P}_{\omega}(\mathbf{r}) d^{3} \mathbf{r}$.

Equations (32) and (35) permit the calculation of the linear response function $\alpha_{\omega}$ and, using Eq. (23), the spectral density $J(\omega)$.

\section{E. Representation of $\alpha_{\omega}$ in terms of a "free energy" difference}

To facilitate the calculation of $\alpha_{\omega}$ it is first noted that each term in Eq. (35) is related to a "free energy" of the dielectric with the dielectric response function $\epsilon_{\omega}(\mathbf{r})$ or $\epsilon_{\text {op }}(\mathbf{r})$ in the external electric field $\mathbf{D}_{12}^{(0)}(\mathbf{r})$. Specifically, the "free energy" $\mathcal{F}_{\omega}$ associated with a charge distribution $\rho_{12}$ interacting with a dielectric with polarization $\mathbf{P}_{\omega}(\mathbf{r})$ and a dielectric response function $\epsilon_{\omega}(\mathbf{r})$, is given by ${ }^{45}$

$$
\begin{aligned}
\mathcal{F}_{\omega} & =\frac{1}{8 \pi} \int \mathbf{D}_{\omega}(\mathbf{r}) \cdot \mathbf{E}_{\omega}(\mathbf{r}) d^{3} \mathbf{r} \\
& =\frac{1}{8 \pi} \int \boldsymbol{\epsilon}_{\omega}(\mathbf{r})\left[\mathbf{E}_{\omega}(\mathbf{r})\right]^{2} d^{3} \mathbf{r} .
\end{aligned}
$$

It can be shown ${ }^{45}$ that this $\mathcal{F}_{\omega}$ equals

$\mathcal{F}_{\omega}=\frac{1}{8 \pi} \int\left[\mathbf{D}_{21}^{(0)}(\mathbf{r})\right]^{2} d^{3} \mathbf{r}-\frac{1}{2} \int \mathbf{P}_{\omega}(\mathbf{r}) \cdot \mathbf{D}_{21}^{(0)}(\mathbf{r}) d^{3} \mathbf{r}$,

and so Eq. (35) can be rewritten as

$$
\alpha_{\omega}=2\left(\mathcal{F}_{\omega}-\mathcal{F}_{\text {op }}\right),
$$

where $\mathcal{F}_{\text {op }}$ is the free energy, Eq. (36), of the charge distribution $\rho_{21}(\mathbf{r})$ in the dielectric environment with the dielectric response function $\epsilon_{\mathrm{op}}(\mathbf{r})$. Equation (38) was derived from molecular considerations and so still represents a molecular statistical mechanical expression, in the long wavelength approximation for treating the solvent.

Equation (38) is a useful generalization in two respects, in frequency domain and in treating the problem quantum mechanically, of a result obtained earlier. ${ }^{31,32}$ The latter related the reorganization energy of the reaction to the (free) energy difference of two hypothetical systems, one of which responds to a difference in charge distributions of the reactant and product states via a static dielectric response and another via an electronic one.

Only the frequencies which correspond to the solvent nuclear motion must be considered, since the solvent electronic motion has been taken into account implicitly in the form of the solvent electronic dielectric response $\epsilon_{\mathrm{op}}(\mathbf{r})$. As a result, the rate constant $k$ of the reaction, Eq. (4), can be rewritten as

$$
\begin{aligned}
k= & \hbar^{-2} \Delta^{2} \int_{-\infty}^{\infty} d t \exp \left[-i t \Delta G^{0} / \hbar-\frac{2}{\pi} \int_{0}^{\omega_{\mathrm{op}}} d \omega\right. \\
& \left.\times \frac{\operatorname{Im} \mathcal{F}_{\omega}}{\hbar \omega^{2}} \frac{\cosh (\hbar \beta \omega / 2-i \omega t)-\cosh (\hbar \beta \omega / 2)}{\sinh (\hbar \beta \omega / 2)}\right],
\end{aligned}
$$


where the standard units, in which $\hbar \neq 1$, have been used. The frequency $\omega_{\text {op }}$ corresponds to the transparency region which separates the frequency region of the solvent nuclear motion and the one of the solvent electronic motion.

The above expression for the rate, Eq. (39), can be considerably simplified by estimating the time-integral in Eq. (39) using a saddle point method ${ }^{16,47}$ and by dividing the whole frequency range of integration into two regions: ${ }^{10,48}$ the low frequency region corresponding to $\omega<4 k_{B} T / \hbar$ and the high frequency region with $\omega>4 k_{B} T / \hbar{ }^{48} \mathrm{We}$ leave this calculation for a future work.

\section{F. Specific solute models}

Calculations based on $\mathcal{F}_{\omega}$ can be used for realistic solute charge distributions, i.e., those not containing point charges. In some idealized models based on point charges, Eq. (36) would yield an infinity which cancels when $\mathcal{F}_{\omega}-\mathcal{F}_{\text {op }}$ is calculated. To avoid this infinity, it is useful to introduce the energy $\mathcal{F}_{\omega}^{\prime}$ of the "dielectric" in the external electric field $\mathbf{D}_{21}^{(0)}(\mathbf{r})::^{45^{\omega}}$

$$
\begin{aligned}
\mathcal{F}_{\omega}^{\prime} & =\mathcal{F}_{\omega}-\frac{1}{8 \pi} \int\left[\mathbf{D}_{21}^{(0)}(\mathbf{r})\right]^{2} d^{3} \mathbf{r} \\
& =-\frac{1}{2} \int \mathbf{P}_{\omega}(\mathbf{r}) \cdot \mathbf{D}_{21}^{(0)}(\mathbf{r}) d^{3} \mathbf{r} .
\end{aligned}
$$

That is, $\mathcal{F}_{\omega}^{\prime}$ differs from $\mathcal{F}_{\omega}$ only by the frequencyindependent self-interaction term in Eq. (40). When the solute can be modeled with point charges $q_{j}$, the representation $\mathcal{F}_{\omega}^{\prime}$ in terms of an electrostatic potential $\phi(\mathbf{r})$ is convenient, Eq. (40) corresponds to

$$
\mathcal{F}_{\omega}^{\prime}=\frac{1}{2} \sum_{j} q_{j} \phi_{\omega}^{\prime}\left(\mathbf{r}_{j}\right),
$$

where $\phi_{\omega}^{\prime}(\mathbf{r})$ is the part of electrostatic potential created by the solvent polarization with the dielectric function $\epsilon_{\omega}(\mathbf{r})$. In the case when the solute is modeled by a point dipole $\mathbf{d}_{0}$, it is more convenient to express $\mathcal{F}_{\omega}^{\prime}$ in terms of an electric field,

$$
\mathcal{F}_{\omega}^{\prime}=-\frac{1}{2} \mathbf{d}_{0} \mathbf{R}_{\omega},
$$

where $\mathbf{R}$ is the reaction field, equal to $-\nabla \phi_{\omega}^{\prime}(\mathbf{r})$, created by the solvent at the site of the dipole.

Any spatial dependence of $\epsilon_{\omega}$ reflects the structural inhomogeneity of the solvent in the vicinity of the reacting species. In the simplest approximation, the polarizability of the solute is neglected and it is so assumed that $\epsilon_{\omega}(\mathbf{r})=1$ in the region occupied by the reactants. An improved approximation for the electronic polarizability of the solute would be $\epsilon_{c} \simeq 2$ inside the cavity, corresponding approximately to an electronic polarizability of the solvent. ${ }^{41}$ As an example, the donor and acceptor have been modeled as spheres of radii $a$ separated by distance $r_{0}$. The charge distribution $\rho_{21}$ consists of two point charges $q$ ( $q$ is an elementary charge) which are situated at the centers of the spheres. It will also be assumed for simplicity that the donor and acceptor are sufficiently distant from each other, $r_{0} \gg a$, so that their dielectric image effect can be neglected. Then using Eqs. (38) and (41) it can readily be shown that $\alpha_{\omega}$ is

$$
\alpha_{\omega}=2 q^{2}\left(\epsilon_{\mathrm{op}}^{-1}-\epsilon_{\omega}^{-1}\right)\left(a^{-1}-r_{0}^{-1}\right) .
$$

Using this equation, the analytical properties of $\epsilon_{\omega}$, and the expression for the reorganization energy $\lambda$ in terms of the spectral density $J(\omega),{ }^{24,48,49}$

$$
\lambda=\frac{1}{\pi} \int_{0}^{\infty} \frac{J(\omega)}{\omega} d \omega,
$$

the standard expression for $\lambda$ is obtained ${ }^{7}$

$$
\lambda=q^{2}\left(\epsilon_{\mathrm{op}}^{-1}-\epsilon_{0}^{-1}\right)\left(a^{-1}-r_{0}^{-1}\right),
$$

where $\epsilon_{0}$ is the static dielectric constant of the solvent.

The dielectric properties of the solvent enter into Eq. (43) in the form of a factor $1 / \epsilon_{\omega}$. Such a form corresponds to a homogeneous solvent and has been commonly used in the theory of electron transfer reaction rates ${ }^{1,2,14}$ and nonlinear spectroscopy. ${ }^{27}$ This form, however, is modified when account is taken of the inhomogeneity of the system near the solute molecule. ${ }^{50}$

Another example is the Onsager model, ${ }^{51}$ which has been used in the spectroscopy of static and dynamical solvatochromism. ${ }^{52}$ In this model the solute has a spherical shape but the charge distribution is approximated by a dipole $d_{0}$ in the center of a polarizable sphere of radius $a$ and the electronic dielectric constant $\epsilon_{c}$ inside the sphere. The effective dipole which would be seen from outside of such a sphere in vacuum ${ }^{53}$ is $d=3 d_{0} /\left(\epsilon_{c}+2\right)$. The solution of the electrostatic equations for this problem is well known. ${ }^{51,53}$ Using Eq. (42) one then obtains that the $\alpha_{\omega}$ in Eq. (35) in this case is ${ }^{54}$

$$
\alpha_{\omega}=\frac{\left(\epsilon_{c}+2\right)^{2}}{3} \frac{d^{2}}{a^{3}}\left[\frac{1}{2 \epsilon_{\mathrm{op}}+\epsilon_{c}}-\frac{1}{2 \epsilon_{\omega}+\epsilon_{c}}\right] .
$$

Comparing Eqs. (46) and (43) one can see that the factor $1 / \epsilon_{\omega}$ for the homogeneous medium is changed to the factor $1 /\left(2 \epsilon_{\omega}+\epsilon_{c}\right)$ in the Onsager model. This change is a result of an inhomogeneity in the system, i.e., it is due to the presence of the solute cavity in the solvent. It can modify the longitudinal relaxation time in the dynamics of the Stokes shift. ${ }^{33,55}$ Often $\epsilon_{c} \simeq 2$ is used. ${ }^{41}$

\section{DISCUSSION}

Linear response theory has frequently been used to relate the fluctuations of the electromagnetic field in the entire environment, with the dielectric properties of the environment, e.g., as in Refs. 16, 17, and 56. The approach in the present paper is somewhat different and simpler, because attention is focused on the calculation of the fluctuations of only a single variable, the reaction coordinate $X$. In this sense our approach is similar to the one used by Mukamel ${ }^{33}$ and by Fleming and co-workers ${ }^{26}$ to treat the state-specific solvation dynamics of chromophoric molecules excited by a sequence of ultrashort laser pulses. The reaction coordinate $X$, defined in 
Eq. (7) as an energy difference of the system in two electronic states has been used by earlier workers ${ }^{37,57,58}$ in more classical treatments of the rate constant.

Equation (16) formally coincides with the correlation function of the collection of molecular harmonic oscillators if one introduces for $J(\omega)$ the spectral density of their normal modes ${ }^{49,59}$ which justifies the usage of this title (spectral density) for $J(\omega)$. The spectral density of the harmonic system is temperature-independent. For real nonlinear systems the spectral density was found to depend strongly on the temperature for low frequency component of $J(\omega)$ ascribed to diffusive motion of the solvent molecules. ${ }^{24}$ It is worthwhile noting also that while for a harmonic oscillator solvent the spectral density is, of course, the same in classical and quantum mechanics, classical and quantum mechanics will give different results for $J(\omega)$ of a real nonlinear solvent.

The spectral density which enters into Eq. (16) can be readily rewritten as a Fourier transform of the imaginary part of the correlation function $C(t)$ :

$$
J(\omega)=2 \int_{0}^{\infty} d t \operatorname{Im}[C(t)] \sin \omega t .
$$

Using this equation as a definition of a spectral density of effective harmonic oscillators a real anharmonic system could be mapped, if one wished, onto the harmonic oscillator model. ${ }^{26,30}$ The present considerations show that the results obtained in the framework of such a model would be valid in the second-order cumulant expansion approximation.

In the present paper we considered electron transfer reactions. Our considerations, however, are applicable to other nonadiabatic processes in a polar environment in which the change of a charge distribution occurs in the process of a nonadiabatic transition. Mukamel ${ }^{30}$ showed in a similar fashion that the correlation function $C(t)$, Eq. (11), enters the final expressions for different order nonlinear optical processes. The present Eqs. (16), (35), and (23) can be used to calculate $J(\omega)$ and $C(t)$ for an appropriate model of a chromophoric solute molecule.

In the dielectric continuum models, such as those in Sec. II F, the inhomogeneity of the system is taken into account in the simplest possible way, namely the solvent is assumed to be homogeneous up to the boundary of the solute cavity with $\epsilon_{\omega}(\mathbf{r})=\epsilon_{\omega}$, which does not depend on $\mathbf{r}$. The last assumption does not take into account molecular nature of the solvent. As a result, one could expect that more realistic $\epsilon_{\omega}(\mathbf{r})$ changes smoothly on a molecular length scale from its bulk value inside the solvent to $\epsilon_{c}$ at the solute boundary (we neglect polarizability of the solute). One approximate way of including molecular effects is given in Ref. 23. As a possible way of dealing with this problem, the mean spherical approximation $^{60-63}$ may be applied. The present treatment also includes an excluded volume effect as discussed in Appendix D and as was discussed earlier by Song et al. ${ }^{23}$

By using Eq. (32) it is assumed that the dielectric response which appears in this equation in terms of the electric susceptibility $\epsilon_{\omega}$ is local in space. This assumption may not be accurate for a field which varies considerably on a molecular scale and one can use a spatially nonlocal dielectric response function ${ }^{18}$ to calculate the electric field in the vicin- ity of the solute. At present, however, only nonlocal spatial dependence (or, equivalently, a $k$-dependence in the Fourier space) of the static electric susceptibility has been estimated, namely from neutron diffraction measurements. ${ }^{64-66}$ Use of the spatially nonlocal dielectric response function for the inhomogeneous solvent would, of course, complicate the solution of Eqs. (32). ${ }^{67}$ The static counterpart of Eq. (38) has been applied to various problems, e.g., Refs. 68 and 69.

\section{ACKNOWLEDGMENTS}

It is a pleasure to acknowledge the support of the National Science Foundation and the Office of Naval Research. One of us (Y.G.) would like to acknowledge the support of the James W. Glanville Postdoctoral Scholarship in Chemistry at Caltech. We would also like to thank Shaul Mukamel, Bruce Berne, and David Chandler for helpful comments.

\section{APPENDIX A: DERIVATION OF EXPRESSION FOR SOLUTE-SOLVENT INTERACTION ENERGY}

Equation (2) describes the electrostatic interaction between the solute characterized by the charge distribution $\rho_{j}(\mathbf{r})$ and the solvent in the particular nuclear configuration, which is characterized by the solvent polarization operator $\hat{\mathbf{P}}_{0}(\mathbf{r})$. To justify it one can calculate the work $W$ which must be done to charge the solute from the hypothetical neutral state with $\rho(\mathbf{r})=0$ to the actual $j$ th state with the charge distribution $\rho(\mathbf{r})=\rho_{j}(\mathbf{r})$ while the solvent nuclear configuration is kept fixed. At any specified solvent nuclear configuration the solvent polarization operator $\hat{\mathbf{P}}_{0}(\mathbf{r})$, which refers to the hypothetical neutral state of the solute, can be viewed as a real function of $\mathbf{r}$ and not only as an operator.

The electronic part of the Hamiltonian operator $H^{\mathrm{el}}$, which acts on the electronic wave function $\Psi$ of the solvent and which describes the solvent interacting with itself and with the charge distribution $\rho(\mathbf{r})$, can be written as

$$
H^{(\mathrm{el})}=H_{0}^{(\mathrm{el})}+\int \rho(\mathbf{r}) \widetilde{\phi}(\mathbf{r}) d^{3} \mathbf{r},
$$

where $\widetilde{\phi}(\mathbf{r})$ is the electrostatic potential. The $\widetilde{\phi}(\mathbf{r})$ in Eq. (A1) must be considered as a quantum mechanical operator (actually, as an operator function parametrically dependent on r) acting on the electronic wave function $\Psi$. The tilde notation is used in this section to denote operators acting on the electronic wave function $\Psi$. In Eq. (A1) $H_{0}^{(\mathrm{el})}$ is an operator which refers to the solvent and includes the interaction of the electrons with the nuclei and with themselves and the kinetic energy operator of the electrons of the solvent. The part of the solute-solvent interaction which is not solute state-specific is also treated as being included in $H_{0}^{(\mathrm{el})} . H_{0}^{(\mathrm{el})}$ does not include the kinetic energy operator for the nuclei, in accord with the Born-Oppenheimer approximation.

The solvent electronic wave function $\Psi$ will change in the process of charging. As a result, the energy of the system $\left\langle\Psi\left|H^{(\mathrm{el})}\right| \Psi\right\rangle$ will change too. To calculate this change one notes that the electrostatic potential $\widetilde{\phi}(\mathbf{r})$ is actually a (functional) derivative of $H^{\mathrm{el}}$ over $\rho$ (r) [cf. Eq. (A1)]. Then, using the Hellman-Feynman theorem on the derivative of the en- 
ergy with respect to a parameter for the system with a parameter-dependent Hamiltonian, ${ }^{70}$ one readily obtains the following expression for the work $W$ to charge the solute in this case,

$$
W=\int d^{3} \mathbf{r} \int_{\rho=0}^{\rho=\rho_{j}} \delta \rho(\mathbf{r}) \hat{\phi}_{\rho}(\mathbf{r})
$$

where $\hat{\phi}_{\rho}(\mathbf{r})=\langle\Psi|\widetilde{\phi}(\mathbf{r})| \Psi\rangle$ is the average of the electrostatic potential $\widetilde{\phi}(\mathbf{r})$ over the ground state electronic wave function $\Psi$ corresponding to the solute charge distribution $\rho(\mathbf{r})$. It is an operator which acts on the nuclear wave function.

The electrostatic potential $\hat{\phi}_{\rho}(\mathbf{r})$, i.e., the average of $\widetilde{\phi}(\mathbf{r})$ over $\Psi$, can be written as a sum of two terms,

$$
\hat{\phi}_{\rho}(\mathbf{r})=\hat{\phi}_{0}(\mathbf{r})+\phi_{\rho}^{\prime}(\mathbf{r}),
$$

where the electrostatic potential $\hat{\phi}_{0}(\mathbf{r})$ corresponds to $\rho(\mathbf{r})$ $=0$ and arises from $\hat{\mathbf{P}}_{\mathbf{0}}(\mathbf{r})$ :

$$
\hat{\phi}_{0}(\mathbf{r})=\int \hat{\mathbf{P}}_{0}\left(\mathbf{r}^{\prime}\right) \cdot \nabla \frac{1}{\left|\mathbf{r}-\mathbf{r}^{\prime}\right|} \mathbf{d}^{3} \mathbf{r}^{\prime} .
$$

The second term in Eq. (A3), $\phi_{\rho}^{\prime}(\mathbf{r})$, describes the additional electronic solvent response to the external electric field corresponding to the charge distribution $\rho(\mathbf{r})$. $\left[\hat{\mathbf{P}}_{0}(\mathbf{r})\right.$ contains the electronic response in the absence of that field.]

As a consequence of Eq. (A3), the work $W$ can be written as

$$
W=W_{0}+W^{\prime},
$$

where $W_{0}$ is the contribution from $\hat{\phi}_{0}(\mathbf{r})$ and $W^{\prime}$ is the contribution from $\phi_{\rho}^{\prime}(\mathbf{r})$. Reexpressing $W_{0}$ in terms of the vacuum electric field $\mathbf{D}_{j}^{(0)}(\mathbf{r})$ and the solvent polarization operator $\hat{\mathbf{P}}_{0}(\mathbf{r})$, using arguments similar to those used in macroscopic electrostatics, ${ }^{45}$ it follows that

$$
W_{0}=\int \rho_{j}(\mathbf{r}) \hat{\phi}_{0}(\mathbf{r}) d^{3} \mathbf{r}=-\int \mathbf{D}_{\mathbf{j}}^{(\mathbf{0})}(\mathbf{r}) \cdot \hat{\mathbf{P}}_{\mathbf{0}}(\mathbf{r}) \mathbf{d}^{3} \mathbf{r},
$$

which is the term denoted by $H_{j}^{\prime}$ in Eq. (2) in the text.

It is usually assumed that the response in $\phi_{\rho}^{\prime}(\mathbf{r})$ depends negligibly on the nuclear configuration of the solvent. As a result, the corresponding contribution $W^{\prime}$ to the work $W$ in Eq. (A2) depends negligibly on the solvent nuclear configuration either and therefore can be added to the solute energy $h_{j}$, renormalizing it. If one wished, one could readily estimate $W^{\prime}$ in terms of the electronic dielectric response function $\epsilon_{\mathrm{op}}(\mathbf{r})$, obtaining as a result, ${ }^{45}$

$$
W^{\prime}=\frac{1}{8 \pi} \int \boldsymbol{\epsilon}_{\mathrm{op}}(\mathbf{r})\left[\mathbf{E}_{j, \mathrm{op}}(\mathbf{r})\right]^{2} d^{3} \mathbf{r}-\frac{1}{8 \pi} \int\left[\mathbf{D}_{j}^{(0)}(\mathbf{r})\right]^{2} d^{3} \mathbf{r},
$$

where $\mathbf{E}_{j, \text { op }}(\mathbf{r})$ is the electric field in the environment with the dielectric response function $\epsilon_{\mathrm{op}}(\mathbf{r}) . \mathbf{E}_{j, \mathrm{op}}(\mathbf{r})$ satisfies Eq. (C3) below, with $\rho_{j}(\mathbf{r})$ replacing $\rho(\mathbf{r}, \mathbf{t})$ in the right-hand side of the first equation in Eq. (C3).

It is useful to relate Eqs. (1) and (2) [also Eq. (A6)] to the insightful molecular treatment of Mandel and Mazur, ${ }^{40}$ and obtain, thereby, the electrostatic contribution to $h_{j}, H_{0}$, and $H_{j}^{\prime}$ in Eq. (1). They obtained Eq. (A8) for the total electric energy of molecules having permanent and induced dipoles (we omit our carets for notational simplicity):

$$
V=\frac{1}{2} \mu^{0} \cdot \mathbf{T} \cdot \boldsymbol{\mu}-\mu \cdot \mathbf{D}^{0}-\frac{1}{2} \mathbf{D}^{\mathbf{0}} \cdot \mathbf{A} \cdot \mathbf{D}^{\mathbf{0}},
$$

where $\boldsymbol{\mu}$ and $\boldsymbol{\mu}^{0}$ are $3 N$-dimensional vectors (whose components in the three-dimensional subspace of solvent molecule $n$ are $\mathbf{d}_{n}$ and $\left.\mathbf{d}_{n}^{0}\right) . \mathbf{D}^{0}$ is a $3 N$-dimensional vector with three components of $\mathbf{D}^{(0)}\left(\mathbf{r}_{n}\right)$ at each molecule $n$ and arises from the solute charges, $\mathbf{T}$ is a symmetric tensor of order $3 N$ $\times 3 N$ with elements $T_{m n}\left[=\nabla_{m} \nabla_{n}\left(1 / r_{m n}\right)\right.$ if $\left.m \neq n\right]$ in threedimensional subspace, and with $T_{m m}=0$. A is a symmetric tensor related to $\mathbf{T}$ by $\mathbf{A}=\alpha \cdot(\mathbf{I}+\alpha \cdot \mathbf{T})^{-\mathbf{1}}$, where $\alpha$ is the polarizability of a solvent molecule and $\mathbf{I}$ is a unit tensor of order $3 N \times 3 N$. [The expression is readily generalized when $\boldsymbol{\alpha}$ is a tensor of order $3 N \times 3 N$, and one obtains the tensorial product $\alpha \cdot(\mathbf{I}+\alpha \cdot \mathbf{T})^{-1}$ for $\mathbf{A}$.] $\nabla_{n}$ is the gradient with respect to the coordinates of molecule $n$.

In Eq. (A8) the second term on the right is equivalent to a term $-\int \hat{\mathbf{P}}_{\mathbf{0}}(\mathbf{r}) \cdot \mathbf{D}^{(0)}(\mathbf{r}) \mathbf{d}^{\mathbf{3}} \mathbf{r}$, when one notes the definition of $\hat{\mathbf{P}}_{0}(\mathbf{r})$ in Eq. (3). The first term in the right is independent of $\mathbf{D}^{(\mathbf{0})}(\mathbf{r})$ and so is the electrostatic contribution to the $H_{0}$ in Eq. (1). The last term in Eq. (A8) does not contain the permanent dipoles $\mathbf{d}_{n}^{0}$ and so is insensitive to nuclear position. It contributes to the $h_{j}$ in Eq. (1).

\section{APPENDIX B: EXPRESSION FOR THE STANDARD FREE ENERGY OF REACTION}

The constant $\Delta G^{0}$ in Eq. (17) is seen from Eqs. (10), (16) and (44) to be

$$
\Delta G^{0}=\langle X\rangle-\lambda .
$$

It can be shown that this $\Delta G^{0}$ coincides with the standard free energy of reaction $\Delta G^{0}$, as follows. The standard free energy of reaction $\Delta G^{0}$ is defined by

$$
\begin{aligned}
e^{-\beta \Delta G^{0}}=\frac{\operatorname{Tr}\left[e^{-\beta H_{2}}\right]}{\operatorname{Tr}\left[e^{-\beta H_{1}}\right]}=\left\langle e_{+}^{-\int_{0}^{\beta} \tilde{X}(\tau) d \tau}\right\rangle, & \\
& \tilde{X}(\tau)=e^{\tau H_{1}} X e^{-\tau H_{1}} .
\end{aligned}
$$

Using the second-order cumulant expansion ${ }^{29}$ one finds from Eq. (B2) that

$$
\begin{aligned}
& \Delta G^{0} \simeq\langle X\rangle-\beta^{-1} \int_{0}^{\beta} d \tau \int_{0}^{\tau} d \tau^{\prime} \widetilde{C}\left(\tau^{\prime}\right), \\
& \widetilde{C}(\tau)=\langle\delta \widetilde{X}(\tau) \delta \widetilde{X}(0)\rangle=C(-i \tau) .
\end{aligned}
$$

Substituting Eq. (16) into Eq. (B3) and integrating over $\tau^{\prime}$ and $\tau$ one finds that $\Delta G^{0}$ in Eq. (B2) is the same as that given by Eq. (B1).

\section{APPENDIX C: MACROSCOPIC POLARIZATION P AND MACROSCOPICAL FIELDS E AND D IN TERMS OF THE MOLECULAR MODEL OF THE SOLVENT}

In this Appendix we relate the average electric field $\mathbf{E}(\mathbf{r}, t)$ and electric displacement $\mathbf{D}(\mathbf{r}, t)$ to the relevant op- 
erators [Eqs. (C2)-(C8)] in terms of our molecular model. We first note that the average polarization $\mathbf{P}(\mathbf{r})$ at $\mathbf{r}$ is given by

$$
\mathbf{P}(\mathbf{r}, t)=\operatorname{Tr}\left[\hat{\mathbf{P}}_{0}(\mathbf{r}) R(t)\right],
$$

where the operator $\hat{\mathbf{P}}_{0}(\mathbf{r})$ of the solvent polarization is defined by Eq. (3) and the time-dependent density matrix $R(t)$ is given by Eq. (19).

We next introduce an electric field operator $\hat{\mathbf{E}}(\mathbf{r}, t)$ as

$$
\hat{\mathbf{E}}(\mathbf{r}, t)=-\nabla \int \hat{\mathbf{P}}_{0}\left(\mathbf{r}^{\prime}\right) \cdot \nabla \frac{1}{\left|\mathbf{r}-\mathbf{r}^{\prime}\right|} d^{3} \mathbf{r}^{\prime}+\mathbf{E}_{\mathrm{op}}(\mathbf{r}, t) .
$$

The first term in Eq. (C2) is an electric field generated by the solvent polarization $\hat{\mathbf{P}}_{\mathbf{0}}(\mathbf{r})$. The second term $\mathbf{E}_{\mathrm{op}}(\mathbf{r}, t)$ is an additional electric field which is due to the external charge and to the instantaneous electronic response of the solvent to that external charge distribution $\rho(\mathbf{r}, t)$. (See also the discussion in Appendix A.) The electric field $\mathbf{E}_{\mathrm{op}}(\mathbf{r}, t)$, which is assumed to be independent on the solvent nuclear configuration, satisfies the equations:

$$
\begin{aligned}
& \nabla \cdot \mathbf{D}_{\mathrm{op}}(\mathbf{r}, t)=4 \pi \rho(\mathbf{r}, t), \quad \nabla \times \mathbf{E}_{\mathrm{op}}(\mathbf{r}, t)=0, \\
& \mathbf{D}_{\mathrm{op}}(\mathbf{r}, t)=\epsilon_{\mathrm{op}}(\mathbf{r}) \mathbf{E}_{\mathrm{op}}(\mathbf{r}, t),
\end{aligned}
$$

where $\rho(\mathbf{r}, t)$ is defined in Eq. (28), $\epsilon_{\mathrm{op}}(\mathbf{r})$ is the electronic dielectric susceptibility of the solvent at $\mathbf{r}$, and $\mathbf{D}_{\mathrm{op}}(\mathbf{r}, t)$ is the electronic electric displacement associated with $\rho(\mathbf{r}, t)$.

We note that the operator $\hat{\mathbf{P}}(\mathbf{r}, t)$ of the total solvent polarization can be written as a sum of two terms

$$
\hat{\mathbf{P}}(\mathbf{r}, t)=\hat{\mathbf{P}}_{\mathbf{0}}(\mathbf{r})+\mathbf{P}_{\mathrm{op}}(\mathbf{r}, t) .
$$

The first term $\hat{\mathbf{P}}_{\mathbf{0}}(\mathbf{r})$ is the operator of the molecular solvent polarization $\hat{\mathbf{P}}_{\mathbf{0}}(\mathbf{r})$, Eq. (3), which corresponds to a hypothetical neutral state of the solute with $\rho(\mathbf{r})=0 . \hat{\mathbf{P}}_{\mathbf{0}}(\mathbf{r})$ itself consists of two parts, one part arising from all the unperturbed dipole moments of the solvent molecules, and the second part being a collective electronic response to the first part [cf. the discussion after Eq. (3)].

The second term in Eq. (C4), $\mathbf{P}_{\text {op }}(\mathbf{r}, t)$, is an additional electronic polarization at fixed positions of the solvent nuclei which arises as a collective response of the solvent electrons to the solute electric field $\mathbf{D}^{(0)}(\mathbf{r}, t)$, Eq. (27). We note that $\mathbf{P}_{\text {op }}(\mathbf{r}, t)$ in Eq. (C4) can be written in the conventional way as

$\mathbf{P}_{\mathrm{op}}(\mathbf{r}, t)=\frac{1}{4 \pi}\left[\mathbf{D}_{\mathrm{op}}(\mathbf{r}, t)-\mathbf{E}_{\mathrm{op}}(\mathbf{r}, t)\right]=\frac{\boldsymbol{\epsilon}_{\mathrm{op}}-1}{4 \pi} \mathbf{E}_{\mathrm{op}}(\mathbf{r}, t)$.

We next define an electric displacement operator $\hat{\mathbf{D}}(\mathbf{r}, t)$ (motivated by the standard macroscopical electrostatic description $^{41,45}$ ) as

$$
\hat{\mathbf{D}}(\mathbf{r}, t)=\hat{\mathbf{E}}(\mathbf{r}, t)+4 \pi \hat{\mathbf{P}}(\mathbf{r}, t),
$$

which can be rewritten using Eqs. (C2), (C4), and (C5) as

$\hat{\mathbf{D}}(\mathbf{r}, t)=\mathbf{D}_{\mathrm{op}}(\mathbf{r}, t)-\nabla \int \hat{\mathbf{P}}_{0}\left(\mathbf{r}^{\prime}\right) \cdot \nabla \frac{1}{\left|\mathbf{r}-\mathbf{r}^{\prime}\right|} d^{3} \mathbf{r}^{\prime}+4 \pi \hat{\mathbf{P}}_{\mathbf{0}}(\mathbf{r})$.
In this equation the first term is an electric displacement which is associated with the solvent electronic response only. The last two terms in Eq. (C7) represent the electric field due to the solvent polarization (the second term) and the solvent polarization operator term itself (the third term).

The average electric field $\mathbf{E}(\mathbf{r}, t)$ and electric displacement $\mathbf{D}(\mathbf{r}, t)$ can then be defined as

$\mathbf{E}(\mathbf{r}, t)=\operatorname{Tr}[\hat{\mathbf{E}}(\mathbf{r}, t) R(t)], \quad \mathbf{D}(\mathbf{r}, t)=\operatorname{Tr}[\hat{\mathbf{D}}(\mathbf{r}, t) R(t)]$,

where, as noted earlier, the time-dependent density matrix $R(t)$ is given by Eq. (19). One could use a bar notation for $\mathbf{E}(\mathbf{r}, t)$ and $\mathbf{D}(\mathbf{r}, t)$, as in Eq. (20), but since they coincide with the conventionally used macroscopic fields, ${ }^{41}$ we omit it. Equation (C8) completes the definition of $\mathbf{E}(\mathbf{r}, t)$ and $\mathbf{D}(\mathbf{r}, t)$ in terms of the operators defined in Eqs. (C2) and (C7) and $\hat{\mathbf{P}}(\mathbf{r})$. They are used in the text in Eqs. (29)-(30).

The Fourier component of the average value of the total polarization per unit $f_{\omega}$ [cf. Eq. (33)],

$$
\mathbf{P}_{\omega}(\mathbf{r})=\frac{1}{f_{\omega}} \int_{-\infty}^{\infty} \operatorname{Tr}[R(t) \hat{\mathbf{P}}(\mathbf{r}, t)] \exp (i \omega t) d t,
$$

is related in a standard way to the corresponding Fourier component of the average electric field [cf. Eq. (32)]

$$
\mathbf{P}_{\omega}(\mathbf{r})=\frac{\epsilon_{\omega}(\mathbf{r})-1}{4 \pi} \mathbf{E}_{\omega}(\mathbf{r}) .
$$

Using Eqs. (C4), (C5), and (C10) one readily obtains Eq. (34).

\section{APPENDIX D: EQUATION FOR THE GENERALIZED SOLVENT SUSCEPTIBILITY $\tilde{\boldsymbol{\chi}}^{(m)}$ IN THE PRESENCE OF A SOLUTE CAVITY}

Two dipolar solvent molecules correlate with each other not only directly via the dipole-dipole interaction between them, but also indirectly via the interaction with other molecules of the solvent. As a result, the correlation between orientations of the two solvent molecules in the vicinity of the solute is modified in comparison with the bulk because of the absence of the fluctuating solvent polarization at the place of the solute. This excluded volume effect leads to modification of the dielectric response of the solvent to the external electric field. ${ }^{23}$ In this Appendix we show how this excluded volume effect naturally appears in the present formulation and demonstrate that the resulting equation, Eq. (D14), coincides with Eq. (3.11) in Ref. 23.

For our purposes, it is convenient to rewrite the electrostatic equations (32) in a different form. We will assume that each term in Eqs. (D1)-(D14) refers to an arbitrary frequency $\omega$ and will omit the index $\omega$ for notational simplicity,

$$
\begin{aligned}
& \mathbf{P}(\mathbf{r})=\chi \mathbf{E}(\mathbf{r}), \\
& \mathbf{E}(\mathbf{r})=\mathbf{D}^{(0)}(\mathbf{r})+\int_{\text {out }} d^{3} \mathbf{r}^{\prime} \mathbf{T}\left(\mathbf{r}-\mathbf{r}^{\prime}\right) \mathbf{P}\left(\mathbf{r}^{\prime}\right),
\end{aligned}
$$

where $\chi=(\epsilon-1) / 4 \pi$ is the dielectric polarizability of the solvent, $\mathbf{D}^{(0)}(\mathbf{r})$ is the external electric field, $\mathbf{E}(\mathbf{r})$ is the local electric field at the point $\mathbf{r}$, and $\mathbf{T}\left(\mathbf{r}-\mathbf{r}^{\prime}\right) \mathbf{P}\left(\mathbf{r}^{\prime}\right)$ is given by Eq. (D2), $\mathbf{T}$ being a tensor: 
$\mathbf{T}\left(\mathbf{r}-\mathbf{r}^{\prime}\right) \mathbf{P}\left(\mathbf{r}^{\prime}\right)=\frac{3 \mathbf{P}\left(\mathbf{r}^{\prime}\right) \cdot\left(\mathbf{r}-\mathbf{r}^{\prime}\right)\left(\mathbf{r}-\mathbf{r}^{\prime}\right)}{\left|\mathbf{r}-\mathbf{r}^{\prime}\right|^{5}}-\frac{\mathbf{P}\left(\mathbf{r}^{\prime}\right)}{\left|\mathbf{r}-\mathbf{r}^{\prime}\right|^{3}}$.

Equation (D2) gives the electric field produced at the point $\mathbf{r}$ by a dipole $\mathbf{P}\left(\mathbf{r}^{\prime}\right)$ at the point $\mathbf{r}^{\prime}$. The integration in Eq. (D1) is over the volume outside of the solute.

Expressing $\mathbf{E}(\mathbf{r})$ in terms of $\mathbf{P}(\mathbf{r})$ from the first part in Eq. (D1) and substituting it into the second part one arrives at the following result relating the solvent polarization $\mathbf{P}(\mathbf{r})$ to the external electric field $\mathbf{D}^{(0)}(\mathbf{r})$ :

$$
\mathbf{D}^{(0)}(\mathbf{r})=\int_{\text {out }} d^{3} \mathbf{r}^{\prime} \tilde{\chi}^{-1}\left(\mathbf{r}-\mathbf{r}^{\prime}\right) \mathbf{P}\left(\mathbf{r}^{\prime}\right),
$$

where $\tilde{\chi}^{-1}\left(\mathbf{r}-\mathbf{r}^{\prime}\right)$ is a tensor, the inverse generalized susceptibility of the bulk solvent in our model. It is defined as

$$
\tilde{\chi}^{-1}\left(\mathbf{r}-\mathbf{r}^{\prime}\right)=\chi^{-1} I \delta^{3}\left(\mathbf{r}-\mathbf{r}^{\prime}\right)-\mathbf{T}\left(\mathbf{r}-\mathbf{r}^{\prime}\right),
$$

where $I$ is a unit tensor. It is convenient to use the following symbolic shorthand notation for Eq. (D4) and subsequent equations:

$$
D_{\text {out }}^{(0)}=\tilde{\chi}_{\text {out }, \text { out }}^{-1} P_{\text {out }},
$$

where the two indices "out" indicate that both $\mathbf{r}$ and $\mathbf{r}^{\prime}$ in the corresponding integral equation are in a region outside the solute cavity. We will also use the index "in" to imply that the corresponding variable is inside the cavity. The notation $\tilde{\chi}_{\text {out,out }}^{-1}$ means $\left(\widetilde{\chi}^{-1}\right)_{\text {out,out }}$ and not $\left(\widetilde{\chi}_{\text {out }, \text { out }}\right)^{-1}$.

In Eq. (D3) both $\mathbf{r}$ and $\mathbf{r}^{\prime}$ are the points outside the solute cavity. We can include $\mathbf{r}$ and $\mathbf{r}^{\prime}$ in the cavity, taking $\mathbf{P}\left(\mathbf{r}^{\prime}\right)=\mathbf{0}$ for $\mathbf{r}^{\prime}$ in the cavity, which in our shorthand notation is simply $P_{\text {in }}=0$, and treating $\mathbf{r}$ as being everywhere, and so extend Eq. (D3) to the whole space,

$$
\mathbf{D}^{(0)}(\mathbf{r})=\int_{\text {all space }} d^{3} \mathbf{r}^{\prime} \tilde{\chi}^{-1}\left(\mathbf{r}-\mathbf{r}^{\prime}\right) \mathbf{P}\left(\mathbf{r}^{\prime}\right),
$$

which can be rewritten in a shorthand notation as (the absence of in and out subscripts denotes $\mathbf{r}$ and $\mathbf{r}^{\prime}$ in all space),

$$
D^{(0)}=\tilde{\chi}^{-1} P .
$$

The last equation can be easily solved by the Fourier transform method. The solution can be written in our shorthand notation as

$$
P=\tilde{\chi} D^{(0)} \text {. }
$$

The solvent polarization can be then written as

$$
P_{\text {out }}=\tilde{\chi}_{\text {out }, \text { out }} D_{\text {out }}^{(0)}+\tilde{\chi}_{\text {out,in }} D_{\text {in }}^{(0)} \text {. }
$$

To find $D_{\text {in }}^{(0)}$ it is noted that

$$
0=P_{\text {in }}=\tilde{\chi}_{\text {in,out }} D_{\text {out }}^{(0)}+\tilde{\chi}_{\text {in,in }} D_{\text {in }}^{(0)},
$$

which yields

$$
D_{\text {in }}^{(0)}=-\left(\tilde{\chi}_{\text {in,in }}\right)^{-1} \tilde{\chi}_{\text {in,out }} D_{\text {out }}^{(0)} .
$$

It is useful to note that the operator $\left(\tilde{\chi}_{\text {in,in }}\right)^{-1}$ is the inverse of the operator $\tilde{\chi}_{\text {in,in }}$ and cannot be obtained from $\tilde{\chi}^{-1}$, but that the appropriate integral equation, represented symbolically by

$$
\left(\tilde{\chi}_{\mathrm{in}, \mathrm{in}}\right)^{-1} \tilde{\chi}_{\mathrm{in}, \mathrm{in}}=I_{\mathrm{in}, \mathrm{in}}
$$

must be solved.

Substituting Eq. (D11) into Eq. (D9) one arrives at Eq. (3.11) in Ref. 23, which in our notation is

$$
\begin{aligned}
& P_{\text {out }}=\tilde{\chi}_{\text {out } \text { out }}^{(m)} D_{\text {out }}^{(0)}, \\
& \tilde{\chi}_{\text {out,out }}^{(m)}=\tilde{\chi}_{\text {out,out }}-\tilde{\chi}_{\text {out,in }}\left(\tilde{\chi}_{\text {in,in }}\right)^{-1} \tilde{\chi}_{\text {in,out }}
\end{aligned}
$$

or, in the standard notation, is

$$
\begin{aligned}
\tilde{\chi}^{(m)}\left(\mathbf{r}, \mathbf{r}^{\prime}\right)= & \tilde{\chi}(\mathbf{r}-\mathbf{r})-\iint_{\text {in }} d^{3} \mathbf{r}^{\prime \prime} d^{3} \mathbf{r}^{\prime \prime \prime} \tilde{\chi}\left(\mathbf{r}-\mathbf{r}^{\prime \prime}\right) \\
& \times \tilde{\chi}_{\text {in }}^{-1}\left(\mathbf{r}^{\prime \prime}, \mathbf{r}^{\prime \prime \prime}\right) \tilde{\chi}\left(\mathbf{r}^{\prime \prime \prime}-\mathbf{r}^{\prime}\right) .
\end{aligned}
$$

${ }^{1}$ M. D. Newton and N. Sutin, Annu. Rev. Phys. Chem. 35, 437 (1984).

${ }^{2}$ R. A. Marcus and N. Sutin, Biochim. Biophys. Acta 811, 265 (1985).

${ }^{3}$ P. F. Barbara, T. J. Meyer, and M. A. Ratner, J. Phys. Chem. 100, 13148 (1996).

${ }^{4}$ M. Lax, J. Chem. Phys. 20, 1752 (1952).

${ }^{5}$ R. Kubo and Y. Toyozawa, Prog. Theor. Phys. 13, 160 (1955).

${ }^{6}$ V. G. Levich and R. R. Dogonadze, Dokl. Akad. Nauk SSSR 124, 123 (1959); V. G. Levich, Adv. Electrochem. Electrochem. Eng. 4, 249 (1966)

${ }^{7}$ R. A. Marcus, J. Chem. Phys. 24, 966 (1956).

${ }^{8}$ R. A. Marcus, Can. J. Chem. 37, 155 (1959).

${ }^{9}$ R. A. Marcus, J. Chem. Phys. 43, 679 (1965).

${ }^{10}$ M. A. Vorotyntsev, R. R. Dogonadze, and A. M. Kuznetsov, Dokl. Akad. Nauk SSSR 195, 1135 (1970).

${ }^{11}$ J. Jortner, J. Chem. Phys. 64, 4860 (1976).

${ }^{12}$ J. Ulstrup and J. Jortner, J. Chem. Phys. 63, 4358 (1975).

${ }^{13}$ N. R. Kestner, J. Logan, and J. Jortner, J. Phys. Chem. 78, 2148 (1974).

${ }^{14} \mathrm{~J}$. Ulstrup, Charge Transfer Processes in Condensed Media (Springer Verlag, Berlin, 1979).

${ }^{15}$ D. Chandler, in Liquids, Freezing and Glass Transition, edited by J. P. Hansen, D. Levesque, and J. Zinn-Justin (Elsevier Science, Amsterdam, 1991), p. 193.

${ }^{16}$ A. A. Ovchinnikov and M. Y. Ovchinnikova, Sov. Phys. JETP 29, 688 (1969).

${ }^{17}$ A. A. Abrikosov, L. P. Gorkov, and I. Y. Dzyaloshinski, Methods of Quantum Field Theory in Statistical Physics (Dover, New York, 1975).

${ }^{18}$ A. A. Kornyshev, in The Chemical Physics of Solvation, Part A, Vol. 38 of Studies in Physical and Theoretical Chemistry, edited by R. R. Dogonadze, E. Kálmán, A. A. Kornyshev, and J. Ulstrup (Elsevier, Amsterdam, 1985), p. 77

${ }^{19}$ A. A. Kornyshev and J. Ulstrup, Chem. Phys. Lett. 126, 203 (1986).

${ }^{20}$ A. A. Kornyshev, A. M. Kuznetsov, D. K. Phelps, and M. Weaver, J. Chem. Phys. 91, 7159 (1989).

${ }^{21}$ R. R. Dogonadze and A. M. Kuznetsov, Elektrokhimia 7, 763 (1971) [English Translation: Sov. Electrochem. 7, 735 (1971)].

${ }^{22}$ R. R. Dogonadze, A. A. Kornyshev, and A. M. Kuznetsov, Theor. Math. Phys. 15, 4047 (1973).

${ }^{23}$ X. Song, D. Chandler, and R. A. Marcus, J. Phys. Chem. 100, 11954 (1996).

${ }^{24}$ S. A. Passino, Y. Nagasawa, and G. R. Fleming, J. Chem. Phys. 107, 6094 (1997).

${ }^{25}$ Y. Nagasawa, S. A. Passino, T. Joo, and G. R. Fleming, J. Chem. Phys. 106, 4840 (1997).

${ }^{26}$ G. R. Fleming and M. Ado, Annu. Rev. Phys. Chem. 47, 109 (1996).

${ }^{27}$ S. Mukamel, Principles of Nonlinear Optical Spectroscopy (Oxford University Press, New York, 1995).

${ }^{28}$ J. S. Bader and B. J. Berne, J. Chem. Phys. 104, 1293 (1995); J. S. Bader, C. M. Cortis, and B. J. Berne, ibid. 106, 2372 (1997); S. A. Egorov and B. J. Berne, ibid. 107, 6050 (1997); S. A. Egorov, E. Rabani, and B. J. Berne, ibid. 108, 1407 (1998); E. Rabani, S. A. Egorov, and B. J. Berne, ibid. 109, 6379 (1998)

${ }^{29}$ R. Kubo, M. Toda, and N. Hashitsume, Statistical Physics II (Springer, Berlin, 1995); R. Kubo, J. Phys. Soc. Jpn. 17, 1100 (1962).

${ }^{30}$ S. Mukamel, J. Phys. Chem. 89, 1077 (1985).

${ }^{31}$ R. A. Marcus, J. Chem. Phys. 38, 1858 (1963).

${ }^{32}$ R. A. Marcus, J. Chem. Phys. 39, 1734 (1963).

${ }^{33}$ R. F. Loring, Y. J. Yan, and S. Mukamel, J. Chem. Phys. 87, 5840 (1987).

${ }^{34}$ V. Hizhnyakov and I. Tehver, Phys. Status Solidi 21, 755 (1967). 
${ }^{35}$ V. V. Hizhnyakov and I. K. Rebane, Sov. Phys. JETP 47, 463 (1978).

${ }^{36}$ V. V. Hizhnyakov and I. J. Tehver, J. Lumin. 18/19, 673 (1979).

${ }^{37}$ R. A. Marcus, Discuss. Faraday Soc. 29, 21 (1960).

${ }^{38}$ R. R. Dogonadze, A. M. Kuznetsov, and M. A. Vorotyntsev, Phys. Status Solidi B 54, 125 (1972).

${ }^{39}$ S. Efrima and M. Bixon, Chem. Phys. 13, 417 (1976).

${ }^{40}$ M. Mandel and P. Mazur, Physica (Utrecht) 24, 116 (1958).

${ }^{41}$ C. J. F. Böttcher, Theory of Electric Polarization (Elsevier, Amsterdam, 1973), Vol. 1.

${ }^{42}$ A. M. Kuznetsov, Nouv. J. Chim. 5, 427 (1981).

${ }^{43}$ A. A. Belousov, A. M. Kuznetsov, and J. Ulstrup, Chem. Phys. 129, 311 (1989).

${ }^{44}$ There is also a static external electric field $\mathbf{D}_{1}^{(0)}(\mathbf{r}$ created by the charge distribution $\rho_{1}(\mathbf{r})$ in the initial electronic state of the solute. It, however, only changes the equilibrium average value of $X$ rather than $C(t)$ and is not a focus of the present calculation.

${ }^{45}$ L. D. Landau and E. M. Lifshitz, Electrodynamics of Continuous Media (Pergamon, New York, 1984).

${ }^{46}$ In Eqs. (32) and (33) we have made use of the fact that the Fourier component $f_{\omega}$ of the generalized force $f(t)$ enters as a simple factor into the expressions for the corresponding Fourier components of the electric field $\mathrm{E}(\mathbf{r}, t)$ and dielectric displacement $\mathrm{D}(\mathbf{r}, t)$ because of the linearity of Eqs. (29) and (30), cf. also Eq. (28).

${ }^{47}$ P. Siders and R. A. Marcus, J. Am. Chem. Soc. 103, 741 (1981).

${ }^{48}$ X. Song and R. A. Marcus, J. Chem. Phys. 99, 7768 (1993).

${ }^{49}$ Y. Georgievskii, C.-P. Hsu, and R. A. Marcus, J. Chem. Phys. 108, 7356 (1998).

${ }^{50}$ Ovchinnikov and Ochinnilova (Ref. 16) considered the case that the dielectric response function $\epsilon_{\omega}$ always enters in the form of $1 / \epsilon_{\omega}$. They used the electric field in vacuum $\mathbf{D}^{(0)}$ instead of the dielectric displacement $\mathbf{D}_{\omega}$ in Eq. (32), a usage which is only applicable for a homogeneous environment and for some special cases.

${ }^{51}$ L. Onsager, J. Am. Chem. Soc. 58, 1486 (1936).

${ }^{52}$ P. F. Barbara and W. Jarzeba, Adv. Photochem. 15, 1 (1990).

${ }^{53} \mathrm{H}$. Fröhlich, Theory of Dielectrics (Oxford University Press, London, 1949).

${ }^{54}$ To calculate the solvent response function $\alpha_{\alpha}$ it is understood that $\mathbf{R}$ in
Eq. (42) denotes the part of the reaction field which is produced by the solvent with a dielectric response function $\epsilon_{\alpha}$ around the solute sphere. The other part of the field is the dielectric response of the solute sphere itself, which does not depend on $\omega$ and cancels in Eq. (38). The dipole moment which inters into Eq. (42) is a bare dipole moment which should be renormalized, $d=3 d_{0} /\left(\epsilon_{c}+2\right)$. It is readily shown using the results of Ref. 53 that $R=2\left(\epsilon_{\omega}-1\right) d /\left(2 \epsilon_{\omega}+\epsilon_{c}\right) a^{3}$. Substituting this equation into Eq. (42) obtains Eq. (46).

${ }^{55}$ B. Bagchi, D. W. Oxtoby, and G. R. Fleming, Chem. Phys. 86, 257 (1984)

${ }^{56}$ L. D. Landau and E. M. Lifshitz, Statistical Physics II (Pergamon, New York, 1986).

${ }^{57}$ A. Warshel, J. Phys. Chem. 86, 2218 (1982).

${ }^{58}$ R. A. Kuharski, J. S. Bader, D. Chandler, M. Sprik, M. L. Klein, and R. W. Impey, J. Chem. Phys. 89, 3248 (1988).

${ }^{59}$ A. J. Leggett et al., Rev. Mod. Phys. 59, 1 (1987).

${ }^{60}$ P. G. Wolynes, J. Chem. Phys. 86, 5133 (1987).

${ }^{61}$ I. Rips, J. Klafter, and J. Jortner, J. Chem. Phys. 88, 3246 (1988).

${ }^{62}$ I. Rips, J. Klafter, and J. Jortner, J. Chem. Phys. 89, 4288 (1988).

${ }^{63}$ M. L. Horng, J. A. Gardecki, A. Papazyan, and M. Maroncelli, J. Phys. Chem. 99, 17311 (1995).

${ }^{64}$ P. A. Bopp, A. A. Kornyshev, and G. Sutmann, Phys. Rev. Lett. 76, 1280 (1996).

${ }^{65}$ A. K. Soper and J. Turner, Int. J. Mod. Phys. B 7, 3049 (1993).

${ }^{66}$ A. K. Soper, J. Chem. Phys. 101, 6888 (1994).

${ }^{67}$ Another question which arises is that, to our knowledge, apart from Ref. 23 , the theoretical results related to a spatially nonlocal dielectric response, refer, thus far, to a homogeneous environment. The applicability of these results to the inhomogeneous solvent in the vicinity of the solute remains to be explored. A possible way to treat a spatially nonlocal dielectric response in the presence of the solute cavity is described in Ref. 23.

${ }^{68}$ R. A. Marcus, J. Chem. Phys. 43, 1261 (1965).

${ }^{69}$ R. A. Marcus, J. Phys. Chem. 94, 1050 (1990).

${ }^{70}$ L. D. Landau and E. M. Lifshitz, Statistical Physics (Pergamon, New York, 1980) 